\title{
Effects of Driver and Secondary Task Characteristics on Lane Change Test Performance
}

\author{
David Rodrick, Vivek Bhise, and Vaithianathan Jothi \\ University of Michigan-Dearborn, Dearborn, Michigan, USA
}

\begin{abstract}
The main objective of this study was to examine the sensitivity of the Lane Change Test (LCT) as proposed by International Organization of Standardization by evaluating LCT performance between primary and dual-task conditions in simulated driving conditions. The study involved four different secondary tasks that involved tracking, visual search, memory, and data entry, each under two different difficulty levels. The primary task involved a series of lane changes on a three-lane straight roadway where the actual lane change trajectory was compared with a normative model of the trajectory. Thus, the lane change performance was measured by the mean deviation of the actual driving trajectory from the normative trajectory. Twenty-four participants within three age groups $(25-34,35-45$, and $>55$ years) and equally distributed between male and female took part in the study. Thus, the study also investigated the effect of age and gender on driving performance. The results showed that secondary tasks that require visual attention and psychomotor coordination deteriorated driving performance the most, whereas tasks that required memory scanning and utilization of the auditory modality least affected driving performance. The study also found differences in LCT performances with respect to three different age categories and gender. $\odot 2012$ Wiley Periodicals, Inc.
\end{abstract}

Keywords: Lane Change Test (LCT); Driving; Distraction; Aging; Secondary task

\section{INTRODUCTION}

Driving can be considered a perceptual-motor control task that requires a driver's physical and/or mental resources to execute different kinds of driving tasks. With the advent of technologies, drivers often engage in various secondary tasks besides driving (e.g., use of in-vehicle information systems, cell phones). During these dual-task conditions, drivers often get distracted, and a safe maintenance of lateral and longitudinal vehicle control is interrupted. Driver distraction has been considered a serious problem that is supported by National Highway Traffic Safety Administration

Correspondence to: David Roddick, 2231 Engineering Complex, 6901 Evergreen Road, Dearborn, MI 48128. Phone: 1-313-593-0326; e-mail: rodrick@umd.umich.edu

Received: 9 November 2010; revised 14 March 2011; accepted 13 April 2011

View this article online at wileyonlinelibrary.com/journal/hfm DOI: $10.1002 / \mathrm{hfm} .20342$
(NHTSA, 2009) data: 1) According to Fatality Analysis Reporting System data, approximately $16 \%$ of all fatal crashes in 2008 involved driver distraction; 2) with respect to age group, the greatest proportion of distracted drivers were younger than 20 and approximately $16 \%$ of all fatal crashes in this age group accounted for distracted driving; and 3) National Motor Vehicle Crash Causation Survey data estimated that approximately $18 \%$ crashes involved distracted driving as the critical reason for crashes.

An earlier study by Stutts et al. (2005) found that young drivers (younger than 20 years) were the most likely to be involved in distraction-related crashes. Furthermore, certain types of distractions, such as adjusting the radio, cassette or $\mathrm{CD}$, were more prominent in drivers younger than 20 years while other vehicle occupants (e.g., young children) were major distractions for drivers between 20 and 29 years, and objects and events outside the vehicle were key distractions for drivers 65 years old or older. The study also found no 
such pronounced difference between male and female drivers, although men were slightly more likely than women to be categorized as distracted at the time of their crash.

As reported by Ramney (2008), there is no generally accepted definition of driver distraction (Trezise et al., 2006). Driver distraction has been defined as "attention given to non-driving related activity, typically to the detriment of driving performance" (Pettitt, Burnett, \& Stevens, 2005), a form of inattentive state during which the driver "is delayed in the recognition of information needed to safely accomplish the driving task because some event, activity, object, or person within or outside the vehicle compels or induces the driver's shifting attention away from the driving task" (Stutts, Reinfurt, Staplin, \& Rodgman, 2001), and a "voluntary or involuntary diversion of attention from the primary driving tasks... reduces a driver's situational awareness, decision making, and/or performance..." (Australian Road Safety Board, 2006, reported in Trezise et al., 2006).

The NHTSA has identified two possible sources of driver distraction. One is distraction caused by electronic or technological sources, such as cell phones or navigation or other infotainment system. The other is more of conventional one (e.g., eating, reading, searching for objects, or interacting with passengers). According to the NHTSA, a driver's distraction can be categorized into visual, manual, and cognitive distraction. Regardless of the type of distraction, studies over the years have demonstrated that a distraction interrupts a driver's performance and safe behavior on the road (Horberry, Anderson, Regan, Triggs, \& Brown, 2006; Neyens \& Boyle, 2008; Strayer \& Drews, 2007). Besides a lack of generally agreed upon definition, there is a debate regarding magnitude of performance degradation due to various forms of interference. With the assumption that a secondary task will interfere with the primary driving task and that driving is a cognitivemotor task, according to Wickens's (2002) multiple resource theory, a secondary task that competes with the primary task for the same cognitive, perceptual, and motor resources will cause greater interference and greater degradation in the driving performance than will those secondary tasks that compete for a single resource (e.g., auditory or cognitive). A few studies support this theory (Engström \& Markkulal, 2007; Hurwitz \& Wheatley, 2002; Tijerina, Parmer, \& Goodman, 1998). According to Wickens's (2002) theory, task interference may occur when both primary and secondary task demands are relatively high, even though they do not compete for the same resources. The classic example of such distraction is the use of mobile phones while driving (e.g., Horrey \& Wickens, 2006; Matthews, Legg, \& Charlton, 2003; Strayer \& Drews, 2004, 2007).

Numerous study findings and national accident data induced the NHTSA to affirm that driver distraction is "a significant and difficult safety problem to address" (NHTSA, 2010). There is a significant effort at the national level to address this problem. Most worthwhile are two comprehensive reviews of literature that aimed to explore the current state of knowledge of driver distraction (Ramney, 2008; Young \& Regan, 2007).

To systematically and internationally tackle the problem, automotive manufacturers identified a need for a low-fidelity standardized driving simulation that resulted in an international published standard (ISO 26022:2010 - Simulated lane change test to assess invehicle secondary task demand). This standard describes a dynamic dual-task method that quantitatively measures human performance degradation on a primary driving-like task while a secondary task is being performed. The result is an estimate of secondary task demand. The method adopted by the standard is laboratory based. The standard defines the method, the minimum requirements for equipment to support the method, and procedures for collecting and analyzing data derived from the method. According to the standard abstract, the method is applicable to all types of interactions with in-vehicle information, communication, entertainment, and control systems; manual, visual, haptic, auditory, and combinations thereof. Secondary tasks requiring speed variations to be performed cannot be tested with this method. It applies to both Original Equipment Manufacturer and aftermarket in-vehicle systems. It also applies to systems either portable or integrated into the vehicle. The driver behavior principles, the specific task procedures, and driving task correspond only to the operation of a passenger car.

While the standard was in draft level, results from various previous studies showed that the Lane Change Test (LCT) is a good representative measure of driving performance (Burns, Trbovich, McCurdie, \& Harbluk, 2005; Mattes \& Hallen, 2008; Wynn \& Richardson, 2008). During the LCT, the primary driving performance measure is called the mean lateral deviation (mdev). Recently, a few authors proposed some additional measures, such as task duration and lane change 
initiation time (e.g., see Harbluk, Burns, Lochner, \& Trbovich, 2007).

In accordance with this ISO working draft, this study used four secondary tasks: 1) critical tracking task (CTT), 2) visual search task (surrogate reference task; SuRT), 3) Sternberg auditory memory task (COTA), and 4) navigation task. Thus, the study aimed to accomplish the following objectives: 1) to examine the sensitivity of the LCT as measured by the primary task performance, 2) to investigate effects age and gender on primary task performance, and 3) to investigate the effects of secondary task performances on the primary task performance.

\section{METHODS}

\subsection{Participants}

The experiment included 24 participants (12 men and 12 women) who held a valid driver's license. The participants were randomly chosen within three age groups: 1) 25 to 34 years, 2) 35 to 45 years, and 3) 55 years or older. There were eight participants in each age group and four in each age and gender combination.

\subsection{Equipment}

\subsubsection{The Driving Simulator}

As referred in the ISO 26022 working draft standard, the study used a PC-based driving simulation program, a 21-inch LCD monitor, a steering wheel, and a gas pedal as the driving simulator. According to the standard, the screen settings were as follows: 1 ) The screen resolution was set at $1280 \times 800$ pixels with a color depth of 32 bits; 2) the horizontal viewing angle to the display for the road scenery was maintained between 45 and 55 degrees; 3 ) the distance between eyes to the display was maintained at between 60 and $65 \mathrm{~cm}$; and 4) the horizon of the visual scene was within -5 and +5 degrees from the participant's eye point height.

A Logitech (Frement, CA) WingMan Formula GP steering wheel was used for lateral maneuvering of the vehicle. The tangential force to turn the steering wheel was less than $20 \mathrm{~N}$. The resolution of the steering wheel was better than 1.5 degrees. As per the standard, the vehicle made a 360 degree turn at a constant speed of $60 \mathrm{~km} / \mathrm{h}$ between 15 and 17 seconds, with the turn diameter being within 70 to 90 meters. The steering wheel was located in front of the simulation monitor, and it was verified that it had no interference with the visual scene of the simulation.

The vehicle's motion was controlled by a gas pedal. The vehicle's maximum speed was $60 \mathrm{~km} / \mathrm{h}$. The participant was instructed to apply the gas pedal to keep the vehicle running at a constant speed of $60 \mathrm{~km} / \mathrm{h}$ throughout the trial. Figure 1 shows the experimental set-up that includes LCT simulator and secondary task stimulus presentation and response devices.

The following sections briefly discuss the devices used for four secondary tasks in this study.

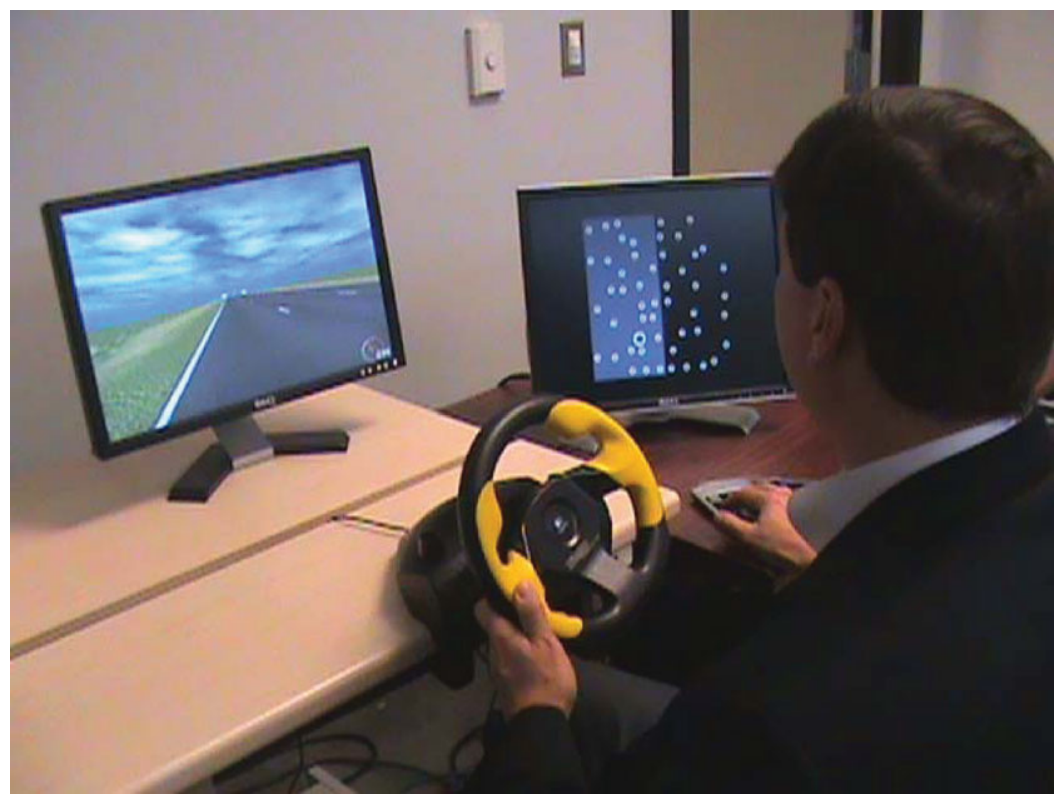

Figure 1 The experimental set-up. 


\subsubsection{CTT}

To present the visual stimuli, a 19 inch flat monitor and a keypad with four arrow keys were used. The up and down arrow keys of the keypad were used to control the movement of the cursor. The display monitor and keypad were placed adjacent to the simulator display unit, to the right side, at approximately $45 \mathrm{~cm}$ from the center of the primary device.

\subsubsection{SURT}

The SuRT uses the same monitor and the keypad described in Section 2.2.2 to present the visual stimuli and to input the response, respectively. For SURT, the left and right arrow keys were used to identify the biggest circle, and the up arrow key was used to confirm the response.

\subsubsection{COTA}

For this task, the study used the audio system of a computer (PC) and a keypad. The PC presented the stimuli through its audio system, and the participant responded orally after which the experimenter recorded the response by using the keypad. The speakers and the keypad were placed away from the participant's visual range.

\subsubsection{Navigation Task}

The TomTom ONE XL (Amsterdam, The Netherlands) global positioning system (GPS) was used for the data entry secondary task. The navigation system has a touch screen to navigate through the system to perform the desired function. The participant interacted with the navigation system through the touch screen. The navigation system was mounted on an adjacent table at approximately $45 \mathrm{~cm}$ to the right side of the simulator display unit.

\subsection{Experimental Task and Corresponding Performance Measures}

\subsubsection{Primary (Driving) Task and Performance Measure}

The primary task involves driving and changing lanes in a simulated environment. In each trial, the participant drove at a constant speed of $60 \mathrm{~km} / \mathrm{h}$, maneuvered lateral positioning of the vehicle by using the steering wheel, and completed a total of 18 lane changes between three lanes based on the instructions from lane change signs. Each course (track) was approximately $3,000 \mathrm{~m}$ long and requires $3 \mathrm{~min}$ of driving time. The lane change signs were placed on both sides of the road and appeared only when the participant was at a distance of $40 \mathrm{~m}$ from the sign. The upward arrow in the lane change sign indicated the lane in which the participant should be driving. The lane changing could have been to the adjacent lane or crossing more than one lane. The mean distance between two successive lane change signs was 150 $\mathrm{m}$. The simulation contained ten different courses wherein the lane change signs appeared randomly. Participants were assigned a course number randomly for any given primary and dual-task condition.

The primary driving task performance was measured by the lateral deviation response. The participant's driving trajectory was collected and compared to the normative trajectory model (theoretical model). The normative trajectory required the participant to change lanes within $10 \mathrm{~m}$ following the sign to change lanes. Then the participant was required to drive in the middle of the instructed lane for the next $20 \mathrm{~m}$, where the lane change sign actually appeared. The primary task performance was the mean difference between the normative model and actual driving, the mdev. Figure 2 shows a snapshot of simulation scenario (a) and a comparison of the normative and actual driving trajectories (b).

\subsubsection{CTT and Performance Measures}

In this secondary task, a horizontal black target line and a center red line was displayed on the secondary task display monitor. When the task was initiated, the black line moved away from the center red line. The movement of this black line was controlled in the vertical direction using the up and down arrow keys of the keypad. The task required the participant to keep the black line as close as possible to the center line by using the arrow keys. The farther the black line from the center line, the faster it moved. If the participant did not react to movements of the black line, it eventually would hit the edge of the display and require stronger input to bring it closer to the center line. At the easy level (defined as 1), the target line would move away from the center red line with a lambda rate of 0.5 . In the hard level (defined as 2), the target line would move 


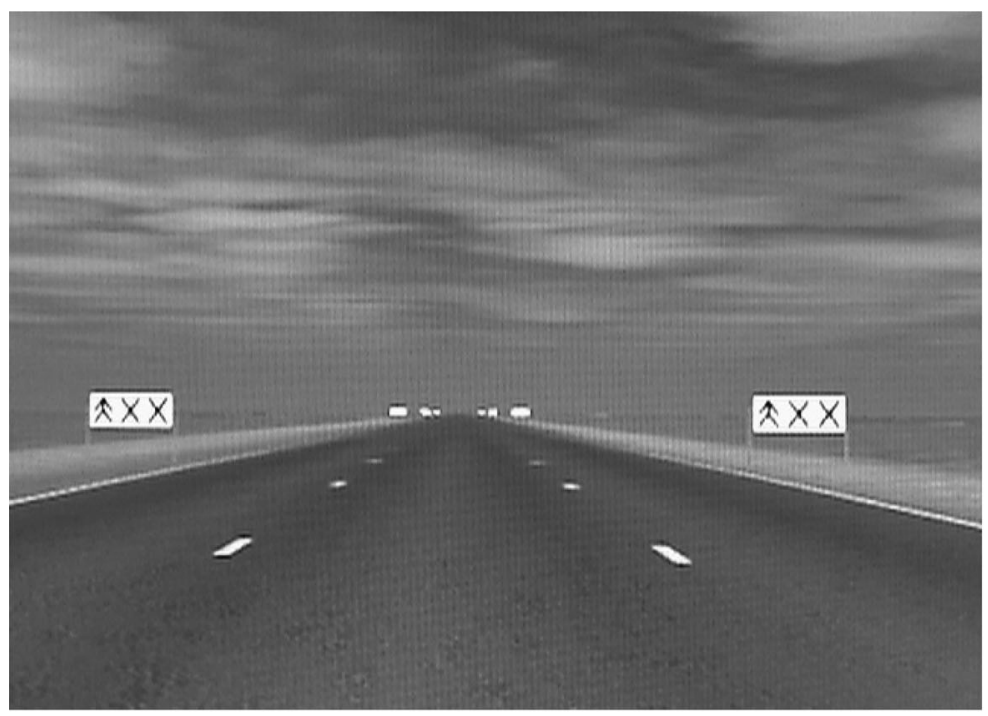

(a)

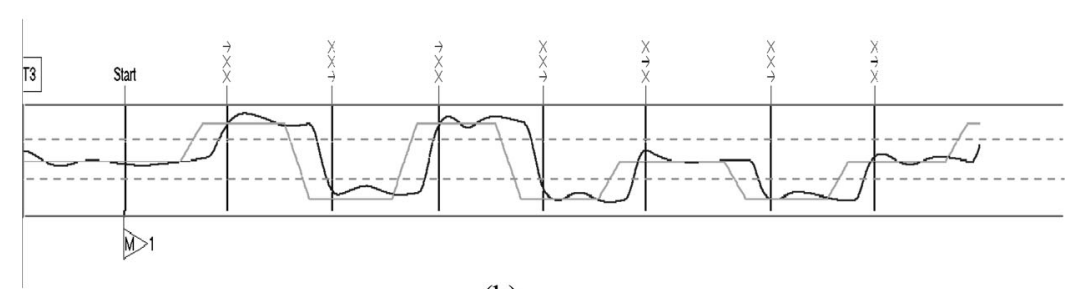

(b)

Figure 2 (a) Lane change signs used in the LCT simulation. (b) Driver's trajectory (dark line) superimposed on the normative model (light line).

faster than in the easy level, where the lambda value was set at 1 .

The performance in this task was measured by two responses: 1) average absolute cursor deviation and 2) percentage of time that the cursor was outside the limit. Average absolute cursor deviation was calculated from the position of the cursor with respect to the center target line at a predefined frequency. The mean of the absolute difference values of the cursor position served as the performance measure. This measure showed how much the participant deviated from the center target throughout the trial. Percentage of time that the cursor was outside the limit was the amount of time the participant was unable to maintain control over the task by having the cursor within the allowed limits. The total time in which the cursor was outside limits was divided by the total task time (approximately $3 \mathrm{~min}$ ). Figure 3 shows the CTT with the target line (dotted line in the middle) and the moving line (solid).

\subsubsection{SuRT and Performance Measure}

In this secondary task, numerous white circles were displayed on the secondary task display unit. The participant had to visually search and identify the target (i.e., the biggest circle) from the left or right array of circles using the left or right arrow key and confirm the response by pressing the up arrow key. Every time the participant confirmed the response, the next set of arrays with circles would appear. The participant was engaged with this task until a full trial of LCT was completed. At level 1 (easy level), there were two arrays of circles, one to the left and one to the right, with numerous circles on each array. Identifying the target or the biggest circle was simple at this level as the target stood out from other circles. At level 2 (hard level) identifying the target was harder and required the participant to pay more attention in searching for the target. In this level, there were eight arrays, four to the left and four to the right, with numerous circles on each array. 


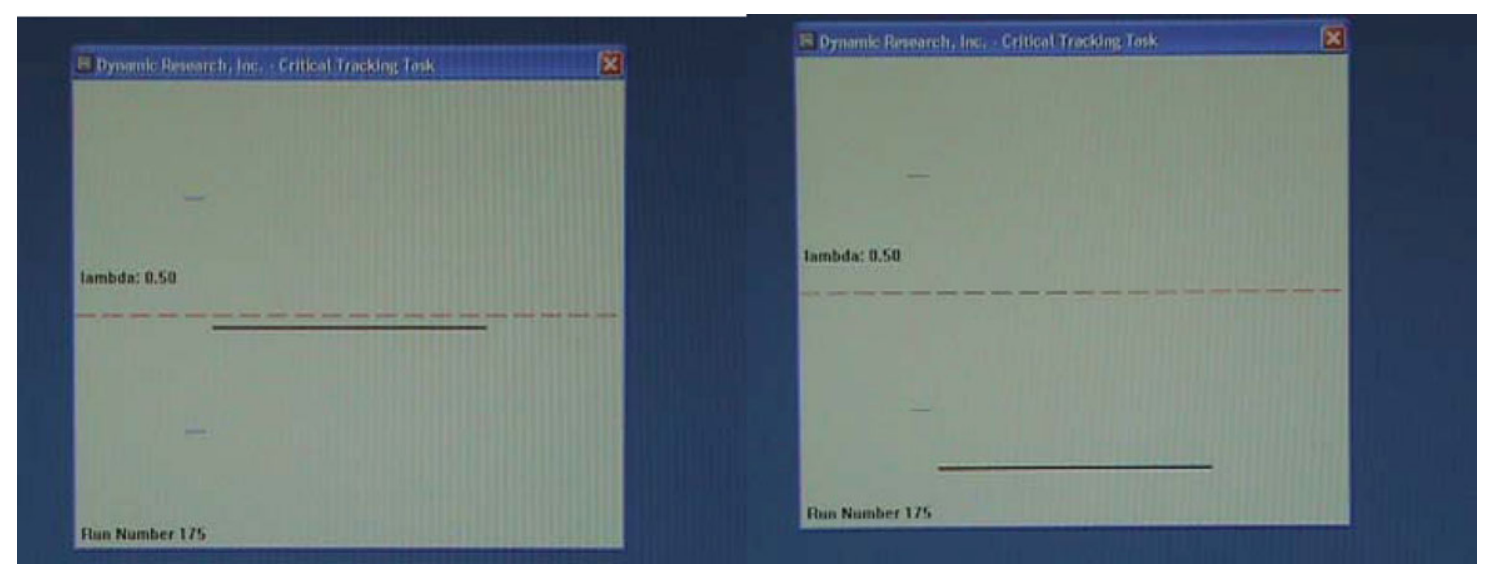

Figure 3 (a) Critical tracking task with cursor (black line) and target (red line). (b) Cursor (black line) moving away from target (red line).

There were three performance measures for this task: 1) Number of items concluded was the number of times the participant attempted to complete the task, irrespective of being correct or wrong; 2) average task completion time for each concluded item was collected, and mean value was calculated as the average task completion time, irrespective of the response being correct or wrong; and 3) percentage of correct responses was calculated by dividing the number of correct responses by the total number of responses. Figure 4 shows the easy and hard levels of the SuRT task with selected arrays of the target circle.

\subsection{4. СОTA and Performance Measure}

In this secondary task, a set of either three (easy level or 1) or six numbers (hard level or 2) were presented in the auditory modality. Following the stimulus presentation, a 15-s break was given, after which a single number was read. The participant's response would be to confirm whether the single number was present in the numbers heard earlier by saying "yes" or "no." The experimenter would then record the participant's response.

The performance measure used in the study for this task was percentage of correct response, which was calculated by dividing the number of correct responses by the total number of responses.

\subsubsection{Navigation (Data Entry) Task and Performance Measures}

This secondary task requires the participant to use a navigation system (TomTom ONE XL) or GPS. At level 1 (easy level) the participant set a preferred volume percentage value as instructed by the experimenter. The preferred volume percentage value was specified before each attempt. A predetermined set of volume values were used for each participant for a given trial. This secondary task at level 1 required a total of 6 steps to complete the task. At level 2 (hard level), participants entered a destination address into the navigation

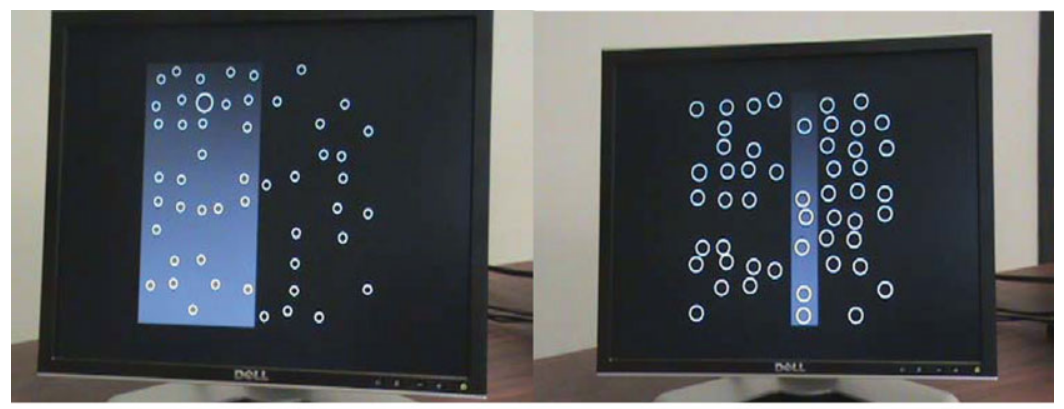

(a)

(b)

Figure 4 Surrogate reference task: (a) easy level and (b) hard level. 


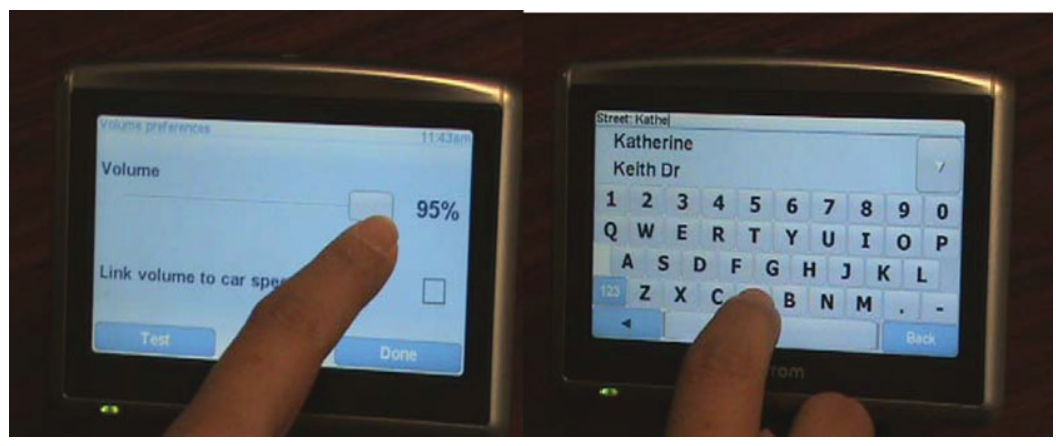

Figure 5 Navigation task: (a) easy level and (b) hard level.

system. The experimenter read aloud the address to the participant before each attempt, then the participant repeated the address to the experimenter to make sure he/she understood it clearly. The participant was allowed to perform the task only if he/she had understood the address clearly. The task required 24 steps to complete the address entry task. Figure 5 shows the easy and hard task response with the navigation system.

There were two performance measures for this task: 1) Number of items concluded was the number of different volume percentage values or destination addresses the participant was able to enter completely in a trial; and 2) average task completion time was the mean value of the times taken to complete a navigation task in a trial. This performance measure was calculated irrespective of the response being correct or wrong.

\subsection{Experimental Design}

The study used a within-subject blocked design in which each participant took part in the same primary, secondary, and dual-task conditions. In each dual-task condition, the participant performed a single secondary task while driving. The independent variables of the study were: 1) task conditions (primary and dual tasks; primary and secondary, four secondary tasks each with two levels of difficulty);2) participant age; and 3) gender. The four secondary tasks, each at two levels (easy and hard), were assigned randomly in a predetermined test sequence that involved measurement of baseline (primary task only) performance at the beginning and end of the test sequence.

\subsection{Procedure}

Upon arrival, the participant completed a consent form, a visual acuity test to assure required score of
$20 / 40$ or better, and a demographic questionnaire. All the necessary instructions for the experiment were prerecorded and played during the experiment.

Following a brief overview of the experiment, the participant began with practice session in the LCT driving simulator to get familiar with the simulation environment until fully satisfied and comfortable with the driving simulator. After adequate practice, the participant took the criterion test in which each participant drove in an identical track twice. If the participant achieved the mdev value of less than $1.20 \mathrm{~m}$, he/she passed the test. If a participant did not achieve this criterion, the experimenter trained him/her over and over until he/she achieved the criterion value. In case a participant was not able to achieve the criterion value after several attempts, the participant was excused from the study.

Upon successful completion of the criterion tests, the participant was given the instruction for the baseline performance during which he/she performed only the primary task. This baseline was marked as the "initial baseline," and the participant performed another baseline at the end, termed "end baseline." Between the initial and end baseline trials, the participant performed on both primary and secondary tasks simultaneously in eight dual-task conditions. Before the dual-task conditions, the participant practiced driving and performing a "dummy" secondary task concurrently to get familiar with dual tasking. Before each dual-task trial the participant was trained on performing a specific secondary task. After training on the specific secondary task, the participant was instructed to perform only the secondary task to get baseline performance data of the secondary task. During any dual-task condition, the participant was not given any instructions on prioritizing between the primary and secondary tasks. The dual-task trial orders were randomized for each participant. 


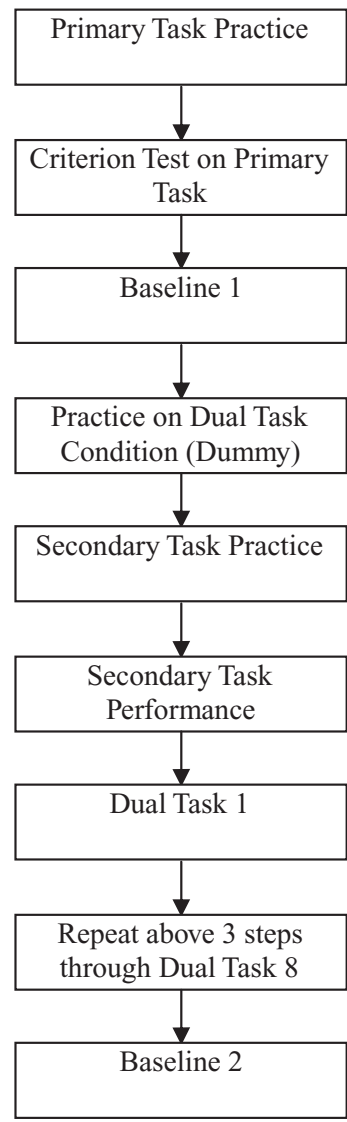

Figure 6 Steps of the experimental procedure.

In each of the trials, the participant was asked maintain the car as close to the driving lane and execute the lane change signs as soon as the lane sign signs appeared on the sides of the roadway. The speed of the vehicle was maintained at $60 \mathrm{~km} / \mathrm{h}$ by asking the driver to floor the gas pedal. To facilitate understanding the experimental procedure, the sequential steps are illustrated in Figure 6.

\section{RESULTS}

\subsection{Primary Task Performance}

The primary task performance measure, mdev, was obtained for two baseline primary task trials and eight dual-task conditions for all the participants. Figure 7 shows the comparison of mdev values for these ten conditions. In this figure, the graphs are shown in four rows and three columns. The baseline data are shown in the left-hand column, and the secondary tasks are presented in the rows.
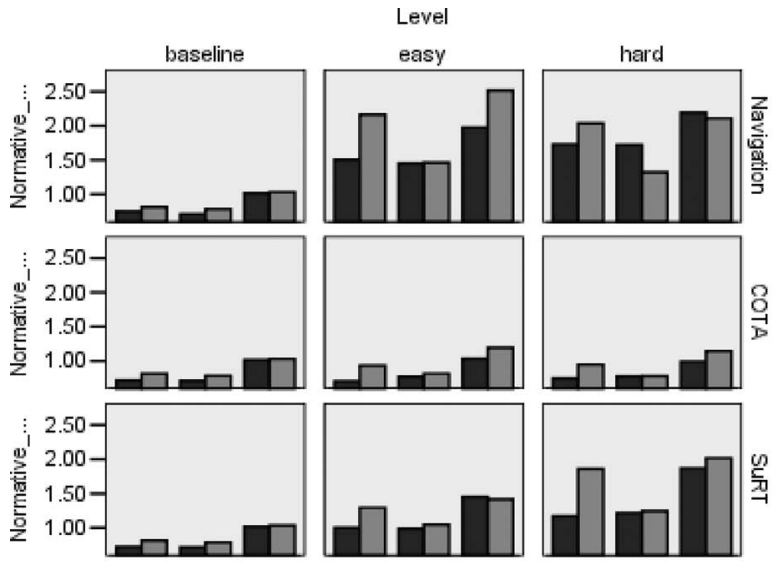

$\overrightarrow{\underline{w^{\prime}}}$
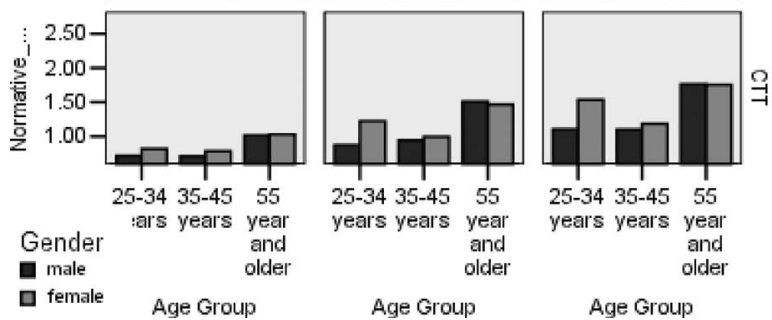

Figure 7 Primary task performance (mdev in meters) by task conditions, task levels, age, and gender.

It can be seen that, except for COTA, the mdev values of LCTs in all other dual-task conditions were higher than those for two primary baseline conditions. A fourway general linear model analysis of variance (ANOVA) was conducted to examine the effects of tasks (four levels - CTT, SURT, COTA, and Navigation), task levels (three levels - primary only, easy, and difficult), age categories (three levels), and gender (two levels) on LCT performance. Table 1 show the summary of the ANOVA.

It can be seen that four different secondary tasks have a significant effect on LCT performance $\left(\mathrm{F}_{3,216}=39.73\right.$, $\mathrm{p}<0.05$ ). The three task levels (primary baseline, secondary easy level, and secondary hard level) also show a significant effect on driving performance $\left(F_{2,216}=\right.$ $71.74, p<.05)$. Three age groups were found to have a significant effect on driving performance $\left(F_{2,216}=\right.$ 40.92, $p<.05$ ).

Finally, the primary task performance also significantly differed depending on gender $\left(F_{1,216}=9.55\right.$, $p<.05)$. The summary table also showed that the interaction effect of age group and gender had a significant effect $\left(F_{2,216}=4.38, p<.05\right)$ over primary task performance. Similarly, the interaction effect of task and task levels had a significant effect on primary task performance $\left(F_{6,216}=11.40, p<.05\right)$. 
TABLE 1. Summary of ANOVA of Primary Task Performance

\begin{tabular}{|c|c|c|c|c|c|}
\hline Source & Sum of Squares & $d f$ & Mean Square & $F$ & $p$ \\
\hline Age Group (1) & 9.779 & 2 & 4.890 & 40.938 & .000 \\
\hline Gender (2) & 1.141 & 1 & 1.141 & 9.552 & .002 \\
\hline Task (3) & 14.236 & 3 & 4.745 & 39.731 & .000 \\
\hline Level (4) & 17.137 & 2 & 8.568 & 71.739 & .000 \\
\hline$(1) *(2)$ & 1.047 & 2 & .523 & 4.381 & .014 \\
\hline$(1) *(3)$ & .532 & 6 & .089 & .743 & .616 \\
\hline$(2) *(3)$ & .019 & 3 & .006 & .052 & .984 \\
\hline$(1) *(2) *(3)$ & .308 & 6 & .051 & .429 & .859 \\
\hline$(1) *(4)$ & .720 & 4 & .180 & 1.508 & .201 \\
\hline$(2) *(4)$ & .209 & 2 & .105 & .877 & .418 \\
\hline$(1) *(2) *(4)$ & .456 & 4 & .114 & .954 & .434 \\
\hline (3) * (4) & 8.167 & 6 & 1.361 & 11.397 & .000 \\
\hline$(1) *(3) *(4)$ & .469 & 12 & .039 & .327 & .984 \\
\hline$(2) *(3) *(4)$ & .699 & 6 & .116 & .975 & .443 \\
\hline$(1) *(2) *(3) *(4)$ & .250 & 12 & .021 & .174 & .999 \\
\hline Error & 25.799 & 216 & .119 & & \\
\hline Total & 496.949 & 288 & & & \\
\hline Corrected Total & 84.333 & 287 & & & \\
\hline
\end{tabular}

Figures 8 and 9 show the interaction effects of age group and gender, and task and task levels on primary task performance, respectively. The estimated marginal means shown are the mean mdev values of primary task performance measures in primary only and two levels of each dual task condition. Subsequent Bonferroni post-hoc tests on the main effects showed that the primary task performances each of age group differed significantly from each other $(p<.05)$, and the mdev was higher for the age group 55 or older, followed by that of 24-34 and 35-45. The post-hoc test also showed significant differences of mdev between CTT and COTA, CTT and Navigation task, SuRT and COTA, SuRT and Navigation task, and COTA and Navigation task. Maximal mdev was highest for Navigation task followed by SuRT, CTT, and COTA. Finally, post-hoc tests showed significant difference of mdev between baseline, easy, and hard levels whereas the highest mdev was found for the hard level, followed by the easy level then baseline. Interestingly there was no significant difference between easy and hard levels of the Navigation task. It can be argued that the two tasks with the navigation system were indeed two distinct tasks and could not be labeled as easy and hard because the easy task required more fine manipulation of psychomotor ability for a longer period of time, whereas the destination entry task required more visual attention on the navigation

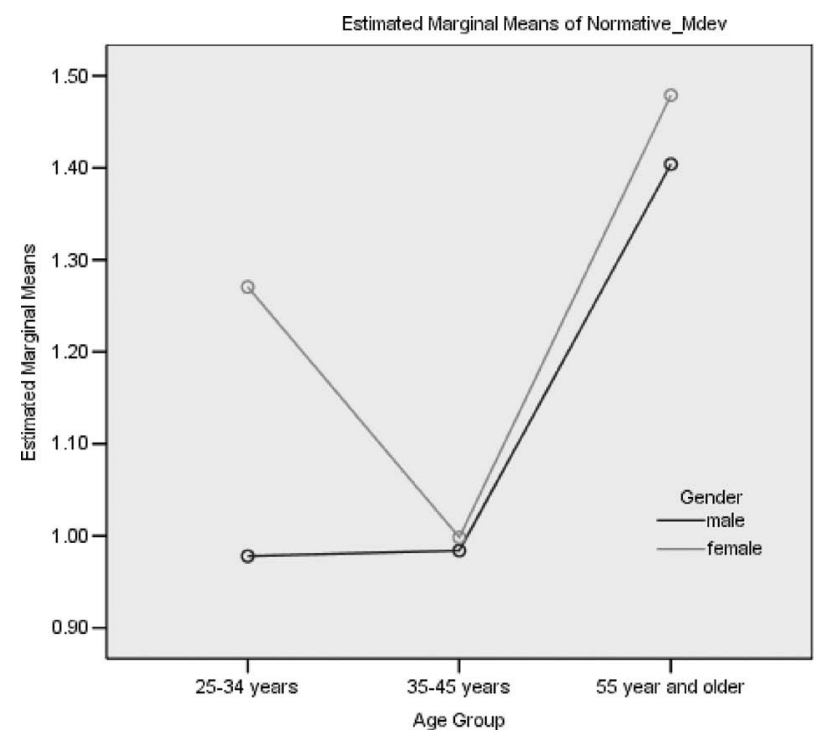

Figure 8 Interaction effect of age group and gender on primary task performance.

device and relatively shorter segments of data entry or psychomotor activity.

\subsection{Secondary Task Performance}

Secondary task performances during dual-task conditions were analyzed using ANOVA. Table 2 shows the results. It can be seen from the table that for each 


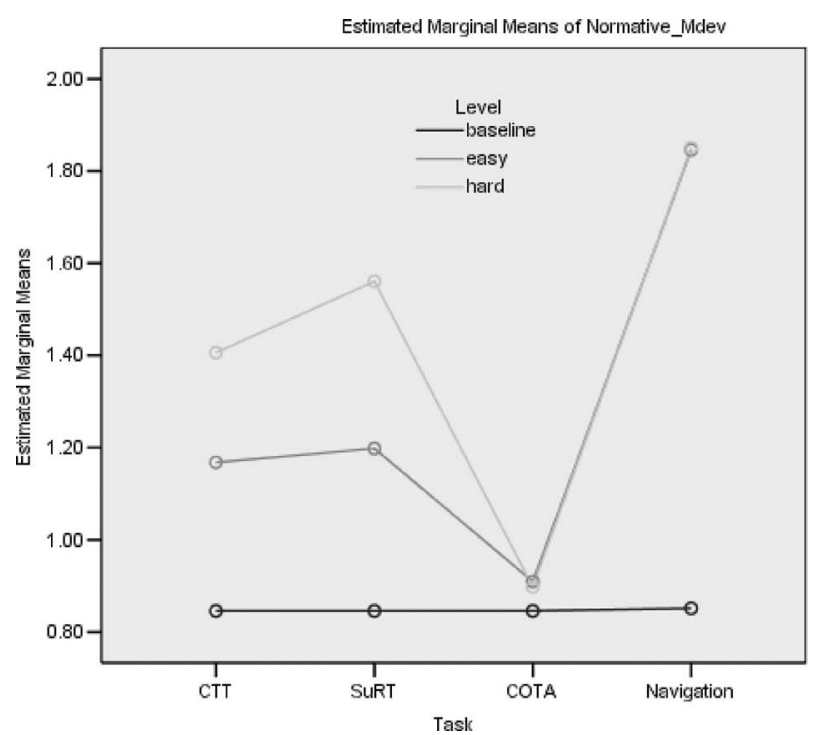

Figure 9 Interaction effect of task and task levels on primary task performance.

performance measure of each secondary task, task level is a significant factor (i.e., performance on easy level was better than that on hard level). In the CTT, there was a significant difference between the age groups 25-34 and 55 or older, and the age group 25-34 performed better in keeping the cursor within the limits. In the SuRT, the age group 25-34 concluded significantly more items than did the age groups $35-45$ and 55 or older.

\section{DISCUSSION}

The study results show that primary task performance as measured by mdev significantly differed between primary and dual-task conditions where mdev value was higher for dual-task condition than in primary task condition. The findings suggest that LCT as a test is sensitive to different task conditions. As the working draft expects, mdev could also be a measure of secondary task workload.

The results showed that older drivers ( 55 years old or older) had the highest mdev (worst performance). The reason for elderly participants performing poorly is possibly the decrement in their psychomotor ability (i.e., to visualize and react physically relatively quickly). In other words, manual and cognitive processing time to respond is slowed down.

Additional analyses conducted to determine differences between the baseline primary task results (beginning and end of the runs) showed that there was no such change in the performance between the two baseline trials. Hence it could be suggested that there was no such detrimental effect toward the end of the experimental session due to fatigue (i.e., experimental length is fairly within the participants' comfort and tolerance level). It can also be argued that the experience in the task had not improved the driving performance as the participant drove several times during the experiment. This argument also assured that the participant had sufficient training before performing in the experiment. Thus, it can be argued that this kind of simulation study could be useful to examine routine tasks that do not require much adaptation time for human participants in a simulated environment.

The driving performance in dual-task conditions was found significantly different for different age groups and gender. Participants in the age group 3545 years performed best. It can be speculated that this group successfully prioritized the primary task while dividing resources in dual-task conditions. Elder participants in the age group 55 years or older had the

TABLE 2. Significant Factors of Secondary Task Performance

\begin{tabular}{llrrr}
\hline Secondary Task & Performance Measure & Significant Factor & F & P \\
\hline \multirow{2}{*}{ Critical Tracking Task } & Average absolute cursor deviation & Task level & 66.04 & .000 \\
& Percentage of Time & Task level & 110.36 & .000 \\
& Cursor outside the Limit & Age & 5.50 & .007 \\
Surrogate Reference Task & Number of Items & Task level & 280.59 & .000 \\
& Concluded & Age & 12.79 & .000 \\
& Average task completion time & Task level & 66.12 & .000 \\
& Percentage of correct response & Task level & 18.01 & .000 \\
Auditory Sternberg Memory Task & Percentage of Correct Responses & Task level & 4.85 & .033 \\
Navigation Task & Number of Items Concluded & Task level & 377.65 & .000 \\
& Average task completion time & Task level & 104.97 & .000 \\
& & & & \\
& & & &
\end{tabular}


worst performance. As the complexity of the task increased, the performance of the elderly participants worsened, and they were more "loaded or stressed" than the younger participants. This finding suggests that older drivers are at higher risk during critical conditions. The chance of elderly participants driving cautiously was totally eliminated by having to drive at a constant speed of $60 \mathrm{~km} / \mathrm{h}$ and constantly changing lanes at equal intervals. Thus, as the task became complex, the older drivers took more time to process information and to coordinate their efforts to respond correctly in a timely manner.

Similar to the baseline results, the male participants performed slightly better than the female participants. The difference in performance measure was consistent across different secondary tasks during dual-task conditions. The reason for the difference in performance due to gender cannot be determined as the information available from the literature is minimal. The primary task (driving) performance is significantly affected by the influence of all the experimental secondary tasks, except COTA. Hence, it is clear that the secondary task that required visual attention and psychomotor coordination had a high impact on driving performance. Among all the secondary tasks, the destination entry task with the navigation system showed significantly higher effects on driving performance. The possible reason for the data entry task being the most distracting task is that it requires more continuous interaction with the device, meaning that fixation on this secondary task and task switching were more than on the other secondary tasks. The navigation device had a touch screen for interface that resulted in higher cognitive demands because it is complex in sensitivity and feedback requires more attention or information acquisition to press the keys. Thus, while the task was switched from primary to secondary, a lane change or lane maintenance was substantially overlooked.

The SuRT at its hard level also showed similar effects, as did data/destination entry task, on driving performance, with respect to the mdev performance measure. The reason for this task being one the most influencing distracters is that it requires a search operation with some strategies to identify the target. Like the navigation system, task switching is rapid and requires a loss of visual attention to the primary task as well as a delay in decision making.

Of all the secondary tasks (not including the COTA) that had significant effects, the CTT had the least effect on driving performance based on the mdev per- formance measure. This secondary task required a constant and continuous effort from the participant throughout the trial. The task pulled visual and manual ability away from the primary task. The probable reasons for this task not being one of the most influencing distractors is that the participant might have paid more attention to the primary task during critical conditions, leaving behind his/her performance in the secondary task. As the tracking task became more difficult to operate, the driving performance worsened due to the cognitive load on the participant. Furthermore, at the hard level, participants had to switch between tasks more often than they did at the easy level.

The COTA at its different levels of three and six number sets did not show significant effects on driving performance. The possible reason for such a case is that the task did not require any visual attention or psychomotor function for recognition (i.e., memorizing a set of numbers did not demand the driver's visual attention or psychomotor resource toward the secondary task).

Thus, from the study, it can be concluded that the secondary tasks that share the required visual attention and psychomotor ability for changing lanes can affect driver performance (slowing to change and/or missing the lane change sign and/or unable to control to stay in the appropriate lane). Thus the results from the present study confirm the results from previous studies that secondary tasks have significant detrimental effects on the primary driving task performance.

It would be beneficial if designers take into account the effects of physical and cognitive resources required for tasks and aging in designing new devices. The key conclusions of this study are that 1 ) visual attention and psychomotor abilities should not be shared by any other secondary tasks for safe and desirable driving behavior on the road, and 2) although auditory perception and memory are not directly part of vehicle control and maneuverability, these resources should be freely allocated to auditory warnings and various regulatory warnings and guiding signs on the road.

The conclusions of the study are summarized as follows:

1. The LCT is highly sensitive to secondary tasks and their respective levels with significantly varying mdev values.

2. The mdev value worsened in the order of high to low, from the Data Entry task to the SuRT to the CTT to the COTA. 
3. Overall, participants in the age range of $>55$ performed poorly compared to the other two age groups in the primary and secondary tasks.

4. Male participants performed the primary task consistently better than the female participants across various dual-task conditions and baseline condition.

One limitation of the study was that the sample size was not large enough for generalization. Although the experiment was highly controlled, we would recommend further investigation instead of generalizing our results. Several implications can be drawn, however, from the study results. First, it appears that male drivers are better at lane changing than are female drivers, at least in this simulated driving test. Although findings on gender effect on performance is inconclusive and whether gender is a causal factor is subject to further investigation, our results indicate that for LCT as a standard, the procedure should recommend an equal amount of male and female drivers in the sample. Practically, gender could be an easier factor to examine with LCT than any other potential causal factors. Thus, from the usability view of LCT, if gender is balanced in the sample, possibly some unnecessary variations can be eliminated from future studies. Second, the decline in perceptual, motor, and cognitive abilities due to aging is such that older drivers performed worse in both primary driving and dual-task conditions. In line with this finding, a practical implication might be to introduce a training regimen for the older driver (e.g., useful field of view training) to improve attention-related performance. Another implication could be an in-vehicle warning system that would warn the driver both in visual and auditory modality when the driver's lane deviation is over some threshold level. Furthermore, LCT simulators could be useful to detect the effect of aging on lane change behavior. Third, as a low-fidelity and robust method, LCT simulation could be a useful tool for both research and training.

\section{References}

Burns, P. C., Trbovich, P. L., McCurdie, T., \& Harbluk, J. L. (2005). Measuring distraction: Task duration and the Lane-Change Test (LCT). Proceedings of the Annual Meeting of the Human Factors and Ergonomics Society, Orlando, FL. September 26-30, 2005. pp. 1980-1983.

Engström, J., \& Markkula, G. (2007). Effects of visual and cognitive task demand on Lane Change Test performance. In Proceedings of the Fourth International
Driving Symposium on Human Factors in Driver Assessment, Training and Vehicle Design. July 9-12, 2007. Stevenson, Washington.

Harbluk, J. L., Burns, P. C., Lochner, M., \& Trbovich, P. L. (2007). Using the Lane Change Test (LCT) to assess distraction: Tests of visual-manual and speech-based operation of navigation system interfaces. In Proceedings of the Fourth International Driving Symposium on Human Factors in Driver Assessment, Training and Vehicle Design, Stevenson, Washington, July 9-12, 2007. pp. 16-22.

Horberry, T., Anderson, J., Regan, M. A., Triggs, T. J., \& Brown, J. (2006). Driver distraction: The effects of concurrent in-vehicle tasks, road environment complexity and age on driving performance. Accident Analysis \& Prevention, 38(1), 185-191.

Horrey, W. J., \& Wickens, C. D. (2006). Examining the impact of cell phone conversations on driving using meta-analytic techniques. Human Factors, 48, 196205.

Hurwitz, J. B., \& Wheatley, D. J. (2002). Using driver performance measures to estimate workload. In Proceedings of the 46th Annual Meeting of the Human Factors and Ergonomics Society. Baltimore: September 30-October 4, 2002.

Mattes, S., \& Hallen, A. (2008). Surrogate distraction measurement techniques: The Lane Change Test. In M. A. Regan, J. D. Lee, \& K. L. Young (Eds.), Driver distraction: Theory, effects and mitigation. Boca Raton, FL: CRC Press.

Matthews, R., Legg, S., \& Charlton, S. (2003). The effect of cell phone type on drivers subjective workload during concurrent driving and conversing. Accident Analysis \& Prevention, 35, 451-457.

National Highway Traffic Safety Administration (NHTSA). (2009). Traffic safety facts (DOT HS 811 216). Washington, DC: NHTSA.

National Highway Traffic Safety Administration (NHTSA). (2010). Driver distraction program (DOT HS 811 299). Washington, DC: NHTSA.

Neyens, D. M., \& Boyle, L. N. (2008). The influence of driver distraction on the severity of injuries sustained by teenage drivers and their passengers. Accident Analysis \& Prevention, 40(1), 254-259.

Pettitt, M., Burnett, G., \& Stevens, A. (2005). Defining driver distraction. Paper presented at World Congress on Intelligent Transport Systems, San Francisco, CA. November 6-10, 2005.

Ramney, T. A. (2008). Driver distraction: A review of the current state-of-knowledge (DOT HS 810 787). Washington, DC: NHTSA.

Strayer, D. L., \& Drews, F. A. (2004). Profiles of driver distraction: Effects of cell phone conversations on younger and older drivers. Human Factors, 46, 640-649. 
Strayer, D. L., \& Drews, F. A. (2007). Cell-phone-induced driver distraction. Current Directions in Psychological Science, 16(3), 128-131.

Stutts, J., Feaganes, J., Reinfurt, D., Rodgman, E., Hamlett, C., Gish, K., \& Staplin, L. (2005). Driver's exposure to distractions in their natural driving environment. Accident Analysis \& Prevention, 37, 1093-1101.

Stutts, J. C., Reinfurt, D. W., Staplin, L., \& Rodgman, E. A. (2001). The role of driver distraction in traffic crashes. Washington, DC: AAA Foundation for Traffic Safety.

Tijerina, L., Parmer, E., \& Goodman, M. J. (1998). Driver workload assessment of route guidance system destination entry while driving: A test track study. Proceedings of the 5th ITS World Congress, Seoul, Korea, CD-ROM. October 12-16, 1990.

Trezise, I., Stoney, E. G., Bishop, B., Eren, J., Harkness, A., Langdon, C., \& Mulder, T. (2006). Report of the road safety committee on the inquiry into driver distraction. Rep. No. 209. Melbourne, Victoria, Australia: Road Safety Committee, Parliament of Victoria.

Wickens, C. D. (2002). Multiple resources and performance prediction. Theoretical Issues in Ergonomics Science, 3, 159-177.

Wynn, T., \& Richardson, J. (2008). Comparison of subjective workload ratings and performance measures of a reference IVIS task. In Proceedings of the European Conference on Human Centred Design for Intelligent Transport Systems, April 3-4, 2008, Lyon, France.

Young, K., \& Regan, M. (2007). Driver distraction: A review of the literature. In I. J. Faulks, M. Regan, M. Stevenson, J. Brown, A. Porter, \& J. D. Irwin (Eds.). Distracted driving. Sydney, NSW: Australasian College of Road Safety, pp. 379-405. 